\title{
DOS ESPECIES NUEVAS DE BURSERA (BURSERACEAE) DE MÉXICO*
}

\author{
Jerzy Rzedowski y Graciela Calderón de Rzedowski \\ Instituto de Ecología, A.C., Centro Regional del Bajío, Apdo. postal 386 \\ 61600 Pátzcuaro, Michoacán, México
}

\section{RESUMEN}

Se describen como nuevas y se ilustran Bursera madrigalii del estado de Michoacán, y B. simplex del estado de Oaxaca, ambas pertenecientes a la sección Bullockia. Las hojas de la primera son similares a las de $B$. hintonii Bullock, pero la morfología de sus órganos reproductores indica un parentesco más cercano con B. xochipalensis Rzed. La segunda se caracteriza por sus hojas simples y al parecer está relacionada con $B$. infernidialis Guevara \& Rzed. y quizás con B. biflora (Rose) Standl.

Palabras clave: Bursera, Burseraceae, México.

\begin{abstract}
Bursera madrigalii, from the state of Michoacán, and B. simplex, from the state of Oaxaca, both belonging to section Bullockia, are described as new and illustrated. The leaves of $B$. madrigalii are similar to those of $B$. hintonii Bullock, but its reproductive morphology indicates a closer relationship with B. xochipalensis Rzed. B. simplex has simple leaves and seems to be related to B. infernidialis Guevara \& Rzed. and perhaps to B. biflora (Rose) Standl.
\end{abstract}

Key words: Bursera, Burseraceae, Mexico.

Se describen a continuación dos especies de Bursera, de cuya existencia ya se tenía noticias desde hace tiempo, pero hubo que esperar a que se avanzara en

\footnotetext{
*Trabajo realizado con apoyo económico del Instituto de Ecología, A. C. (cuenta 902-07), del Consejo Nacional de Ciencia y Tecnología y de la Comisión Nacional para el Conocimiento y Uso de la Biodiversidad.
} 
el conocimiento más completo del género para afirmar su situación de nuevas y sus posibles relaciones de parentesco.

Bursera madrigalii Rzed. \& Calderón sp. nov. Fig. 1

Arbor ut videtur dioecia usque $9 \mathrm{~m}$ alta; truncus cortice griseo non exfolianti; ramuli juniores villosi; foliorum rosulae cataphyllis exterioribus cinctae, folia imparipinnata, rhachidi alata, foliola (5)9-11(15), elliptica ad lanceolata vel oblonga (foliolum terminale saepe rhomboideum), 4-9(11) cm longa, 1-3(4) $\mathrm{cm}$ lata, apice acuta ad acuminata, marginibus crenatis vel crenato-serratis, supra juventute puberula, maturitate glabrescentia, subtus praecipue ad venas puberula; inflorescentiae laxae paniculatae multiflorae usque $20 \mathrm{~cm}$ longae; flores masculi tetrameri, calycis lobi triangulares ca. $1 \mathrm{~mm}$ longi, petala cremea oblonga 2.5-3 $\mathrm{mm}$ longa, stamina 8; flores feminei masculinorum similes; fructus plerumque numerosi, bivalvati, obovoidei, ovoidei vel subsphaerici, 7-10(12) mm longi, pyrenae lenticulares 4$6 \mathrm{~mm}$ longae, pseudoarillo aurantiaco per $1 / 2$ vel $2 / 3$ partibus inferis indutae.

Árbol aparentemente dioico hasta de $9 \mathrm{~m}$ de alto, aromático al estrujarse; tronco hasta de $45 \mathrm{~cm}$ de diámetro, de corteza gris, no exfoliante, rugosa, ramillas rojizas, las más jóvenes vilosas; hojas aglomeradas en rosetas dispuestas en los ápices de ramillas cortas, o bien, alternas en ramillas vigorosas de crecimiento nuevo, precedidas en su aparición por un conjunto de catafilos oblongos a ovados, hasta de $2 \mathrm{~cm}$ de largo, densamente rojizo-tomentosos por fuera, pubérulos o casi glabros por dentro, algo persistentes; peciolos de 4 a $8(10) \mathrm{cm}$ de largo, vilosos, láminas de 12 a 25(30) cm de largo, de 8 a 13(15) cm de ancho, imparipinnadas con (5)9 a 11(15) foliolos sésiles o subsésiles, raquis manifiestamente alado, alas hasta de $3 \mathrm{~mm}$ de ancho en la parte distal de la hoja, más angostas en la proximal, de margen entero o a veces crenado, foliolos laterales elípticos o lanceolados a oblongos, de 4 a 9(11) $\mathrm{cm}$ de largo y de 1 a 3(4) $\mathrm{cm}$ de ancho, el terminal con frecuencia algo romboide y a menudo un poco más largo o un poco más corto que los laterales, ápice agudo a acuminado, base truncada a cuneada, con frecuencia algo oblicua, margen crenado o crenado-serrado, de textura cartácea, con nervaduras muy manifiestas en el envés, verdes oscuros y pubérulos en la juventud, pero tendiendo a glabros en la madurez en el haz, más pálidos y pubérulos principalmente a lo largo de las nervaduras en el envés; inflorescencias en forma de panículas laxas y multifloras, hasta de $20 \mathrm{~cm}$ de largo y $7 \mathrm{~cm}$ de diámetro, con pedúnculos hasta de $6 \mathrm{~cm}$ de largo, vilosos, raquis y ejes secundarios hispídulo-pubérulos con pelos extendidos, brácteas linear-subuladas, de 2 a $6 \mathrm{~mm}$ de largo, precozmente caducas, pedicelos filiformes, hasta de $4 \mathrm{~mm}$ de largo; flores masculinas tetrámeras; lóbulos del cáliz triangulares, de 0.7 a $0.8 \mathrm{~mm}$ de largo, densamente hispídulos por fuera, 
Rzedowski y Calderón de Rzedowski: Dos nuevas especies de Bursera de México

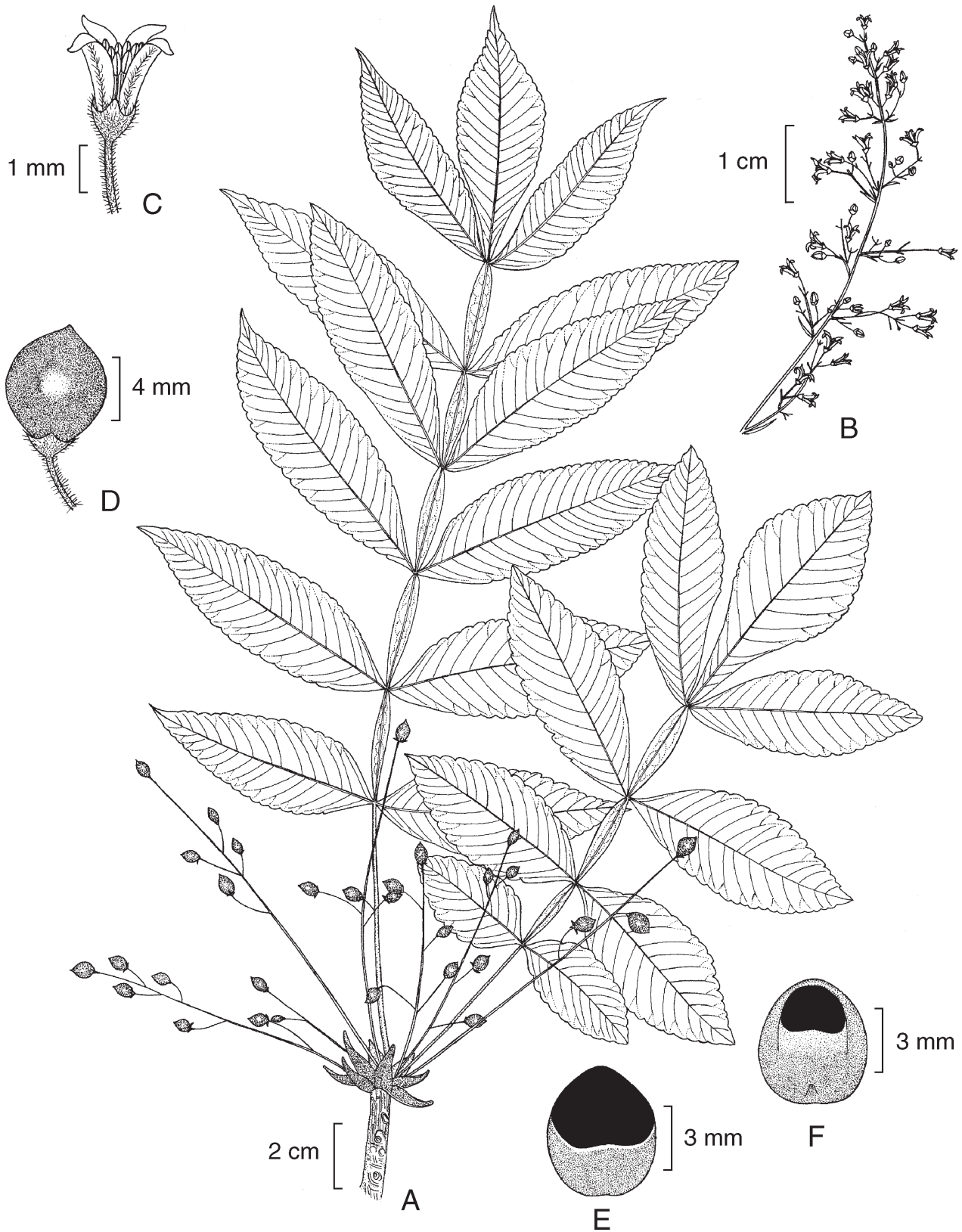

Fig. 1. Bursera madrigalii Rzed. \& Calderón. A. rama con catafilos, hojas y frutos jóvenes; B. trozo de inflorescencia masculina; C. flor masculina; D. fruto maduro; E y F. hueso visto por ambas caras. Ilustrado por Rogelio Cárdenas. 
pétalos oblongos, de 2.5 a $3 \mathrm{~mm}$ de largo, de color crema, hispídulos por fuera a lo largo de la línea media, estambres 8, filamentos de $1 \mathrm{a} 1.2 \mathrm{~mm}$ de largo, anteras angostamente oblongas, de ca. $1 \mathrm{~mm}$ de largo, pistilodio diminuto; flores femeninas (se observaron sólo sus restos) similares a las masculinas, anteras de los estaminodios de ca. $0.5 \mathrm{~mm}$ de largo; frutos a menudo numerosos en la infrutescencia, bivalvados, obovoides u ovoides a subesféricos, puntiagudos, de 7 a 10(12) $\mathrm{mm}$ de largo, rojizos en la madurez, glabros, hueso lenticular, de 4 a $6 \mathrm{~mm}$ de largo y 3 a $7 \mathrm{~mm}$ de ancho, cubierto en la mitad o en los $2 / 3$ inferiores por un pseudoarilo anaranjado, la porción expuesta negra.

Tipo: México, Michoacán: Rancho El Tigre, municipio de Morelia, selva baja caducifolia, alt. 2040 m, 21.VII.1987, X. Madrigal Sánchez 4249 (IEB, isotipos por distribuirse).

Otros materiales examinados: México, Michoacán: Rancho El Tigre, municipio de Morelia, X. Madrigal Sánchez 4250 (IEB); Paso Tendido, ejido Túmbisca, municipio de Morelia, X. Madrigal Sánchez 4259 (IEB); Puerto de Arúmbaro a $12 \mathrm{~km}$ al sur de Temascal, camino a Huetamo, municipio de Tzitzio, E. Martínez Salas y J. C. Soto 1462 (MEXU); ibid., J. C. Soto Núñez y E. M. Martínez Salas 4209 (MEXU); $1 / 4 \mathrm{~km}$ al E de Carrizalillo, municipio de Jungapeo, $S$. Ontiveros A. y P. Vargas H. 110 (IEB); $8 \mathrm{~km}$ al SE de Tzitzio, municipio de Tzitzio, S. Ontiveros Alvarado s. n., XI.2000 (IEB); $15 \mathrm{~km}$ al SW de Zitácuaro, municipio de Benito Juárez, $T$. P. Ramamoorthy 4394 (MEXU); El Tepehuaje, cerca de la escuela, municipio de Tzitzio, M. A. Silva Farías 2 (IEB), s. n., 11.IX.2002 (IEB); al norte de Tepehuaje, municipio de Tzitzio, M. A. Silva Farías 3 (IEB); entre El Tepehuaje y La Escondida, municipio de Tzitzio, M. A. Silva Farías s. n., 12.IX.2002 (IEB); La Igriega, 11 km al SW de Zitácuaro, municipio de Zitácuaro, J. C. Soto Núñez 5270 (MEXU).

Bursera madrigalii se conoce de las estribaciones meridionales del Eje Volcánico Transversal en el norte de Michoacán, donde habita en un intervalo altitudinal de 1550 a $2040 \mathrm{~m}$, que con frecuencia corresponde al ecotono entre el bosque tropical caducifolio y el bosque de encino y/o de pino. Recibe el nombre común de "copal" y en general no se registra como elemento abundante, aunque se le observa con alguna frecuencia en cercas vivas. Florece (probablemente desde mayo) en junio, simultáneamente con la aparición de nuevo follaje.

En virtud de sus flores tetrámeras y fruto bivalvado, pertenece a la sección Bullockia. En la forma y en el tamaño de sus hojas, las plantas de B. madrigalii son similares a las de $B$. hintonii Bullock e inicialmente se identificaron con este último nombre. Sin embargo, las dos especies no parecen estar relacionadas de cerca entre sí, pues difieren en las siguientes características de sus partes reproductoras: 
Rzedowski y Calderón de Rzedowski: Dos nuevas especies de Bursera de México

\begin{tabular}{|l|l|}
\hline \multicolumn{1}{|c|}{ Bursera madrigalii } & \multicolumn{1}{c|}{ Bursera hintonii } \\
\hline $\begin{array}{l}\text { panícula laxa y moderadamente } \\
\text { pubescente }\end{array}$ & $\begin{array}{l}\text { panícula singularmente compacta } \\
\text { y densamente pubescente }\end{array}$ \\
\hline $\begin{array}{l}\text { lóbulos del cáliz más de } 3 \text { veces } \\
\text { más cortos que los pétalos }\end{array}$ & $\begin{array}{l}\text { lóbulos del cáliz de la mitad } \\
\text { del largo de los pétalos }\end{array}$ \\
\hline $\begin{array}{l}\text { hueso de } 4 \text { a } 6 \mathrm{~mm} \text { de largo, } \\
\text { con una notable porción sin } \\
\text { cubrir por el pseudoarilo }\end{array}$ & $\begin{array}{l}\text { hueso de } 8 \text { a } 9 \mathrm{~mm} \text { de } \\
\text { largo, casi totalmente cubierto } \\
\text { por el pseudoarilo }\end{array}$ \\
\hline
\end{tabular}

Además, los tallos y las hojas de B. madrigalii son mucho menos densamente pubescentes que las de $B$. hintonii y aunque tal carácter a veces es difícil de cuantificar, cabe observar que mientras las hojas maduras de esta última planta se sienten suaves al tacto por el envés, tal rasgo no se presenta en la que se describe como nueva.

Es probable entonces que el parentesco más próximo de $B$. madrigalii se encuentra con el grupo de especies de la sección Bullockia que carecen de hueso casi totalmente cubierto por el pseudoarilo y en este conjunto el elemento morfológicamente más parecido es $B$. xochipalensis Rzed., planta conocida de la parte oriental de la cuenca del Balsas, en particular de Guerrero, Oaxaca y Puebla.

Esta última especie coincide también con B. madrigalii en el carácter laxo de la panícula y en los lóbulos del cáliz notablemente cortos. Difiere, sin embargo, en:

\begin{tabular}{|l|l|}
\hline \multicolumn{1}{|c|}{ Bursera xochipalensis } & \multicolumn{1}{c|}{ Bursera madrigalii } \\
\hline $\begin{array}{l}\text { catafilos glabros por fuera y seríceos } \\
\text { por dentro, pronto deciduos }\end{array}$ & $\begin{array}{l}\text { catafilos densamente tomentosos por fuera, } \\
\text { pubérulos o casi glabros por dentro, } \\
\text { moderadamente persistentes }\end{array}$ \\
\hline $\begin{array}{l}\text { hojas con el raquis angostamente alado } \\
\text { o desprovisto de alas }\end{array}$ & hojas con el raquis manifiestamente alado \\
\hline $\begin{array}{l}\text { foliolos (5)7 a 9(11), angostamente } \\
\text { lanceolados a angostamente oblongos }\end{array}$ & $\begin{array}{l}\text { foliolos (5)9 a 11(15), elípticos, } \\
\text { lanceolados u oblongos }\end{array}$ \\
\hline $\begin{array}{l}\text { infrutescencia con reducido número } \\
\text { de frutos }\end{array}$ & infrutescencia con numerosos frutos \\
\hline frutos de 12 a $15 \mathrm{~mm}$ de largo & frutos de 7 a 10(12) mm largo \\
\hline
\end{tabular}

El nombre de la especie está dedicado al M. en C. Xavier Madrigal Sánchez, destacado botánico michoacano, autor de numerosas contribuciones al estudio del universo vegetal del estado, pero especialmente interesado en aspectos del conocimiento, del apropiado aprovechamiento y de la conservación de los bosques. 
En el marco de su trabajo sobre los árboles del municipio de Morelia, Madrigal Sánchez y Guridi Gómez (2002) fueron los primeros en distinguir y mencionar a este particular copal.

Bursera simplex Rzed. \& Calderón sp. nov. Fig. 2

Arbor ut videtur dioecia usque $10 \mathrm{~m}$ alta; truncus cortice griseo non exfolianti; foliorum rosulae cataphyllis exterioribus cinctae, folia simplicia vel rarissime trifoliolata plerumque elliptica, interdum oblonga vel oblanceolata, $2-5 \mathrm{~cm}$ longa, $0.8-$ $2.2 \mathrm{~cm}$ lata, apice rotundata vel obtusa, marginibus regulariter serratis, juventute puberula, maturitate solum ad costam pilibus instructa; inflorescentiae masculae racemosae 1-5(7)-florae; flores masculi tetrameri, calycis lobi triangulares vel anguste triangulares ca. $1 \mathrm{~mm}$ longi, petala cremea oblonga ca. $5 \mathrm{~mm}$ longa; flores feminei plerumque solitarii, aliter terni, masculinorum similes sed petalis saepe $3 \mathrm{~mm}$ longis, ovarium biloculare; fructus bivalvati, ellipsoidei vel obovoidei, 10-13 $\mathrm{mm}$ longi, pyrenae late ellipticae vel suborbiculares aliquantum compressae 6-7 $\mathrm{mm}$ longae, pseudoarillo atrorubro per minus quam $1 / 3$ usque fere $1 / 2$ partibus inferis involutae.

Árbol aparentemente dioico, resinoso, aromático al estrujarse, hasta de 10 $\mathrm{m}$ de alto; tronco hasta de $35 \mathrm{~cm}$ de diámetro, de corteza gris, no exfoliante, lisa, ramillas rojizas oscuras, glabras; hojas aglomeradas en rosetas ubicadas en los ápices de las ramillas, precedidas en su aparición por varios catafilos dispuestos esencialmente en dos series, los exteriores de forma deltoide, de ca. $2 \mathrm{~mm}$ de largo, de color rojo oscuro, glabros en ambas superficies pero con frecuencia ciliados en el margen, los interiores oblongos, de 5 a $8 \mathrm{~mm}$ de largo, apiculados, de color café, glabros en la mayor parte de su superficie, pero a menudo vilosos hacia el ápice, todos pronto caedizos; peciolos de 3 a $12 \mathrm{~mm}$ de largo, pilósulos a glabros, láminas foliares simples o por excepción alguna trifoliolada, mayormente elípticas, variando a oblongas u oblanceoladas, de 2 a $5 \mathrm{~cm}$ de largo, de 0.8 a $2.2 \mathrm{~cm}$ de ancho, redondeadas u obtusas en el ápice, cuneadas a redondeadas en la base, más o menos regularmente serradas en el margen con los dientes redondeados, de textura cartácea en la madurez, nerviación prominente en el envés, verdes oscuras, muy pronto glabras y algo brillantes en el haz, un poco más pálidas en el envés, pubérulas en la juventud en el envés, en la madurez con la pubescencia casi ausente o principalmente restringida a la costa; inflorescencias masculinas en forma de racimos hasta de $4 \mathrm{~cm}$ de largo, llevando 1 a 5(7) flores, bracteolas lineares, hasta de $2 \mathrm{~mm}$ de largo, precozmente caducas, pedicelos filiformes, hasta de $6 \mathrm{~mm}$ de largo, esparcidamente pilósulos, lóbulos del cáliz 4, triangulares a angostamente triangulares, de ca. $1 \mathrm{~mm}$ de largo, pilósulos por fuera, pétalos 4 , de color crema, oblongos, de ca. $5 \mathrm{~mm}$ de largo, glabros o con pocos pelos por fuera a lo largo de 
Rzedowski y Calderón de Rzedowski: Dos nuevas especies de Bursera de México

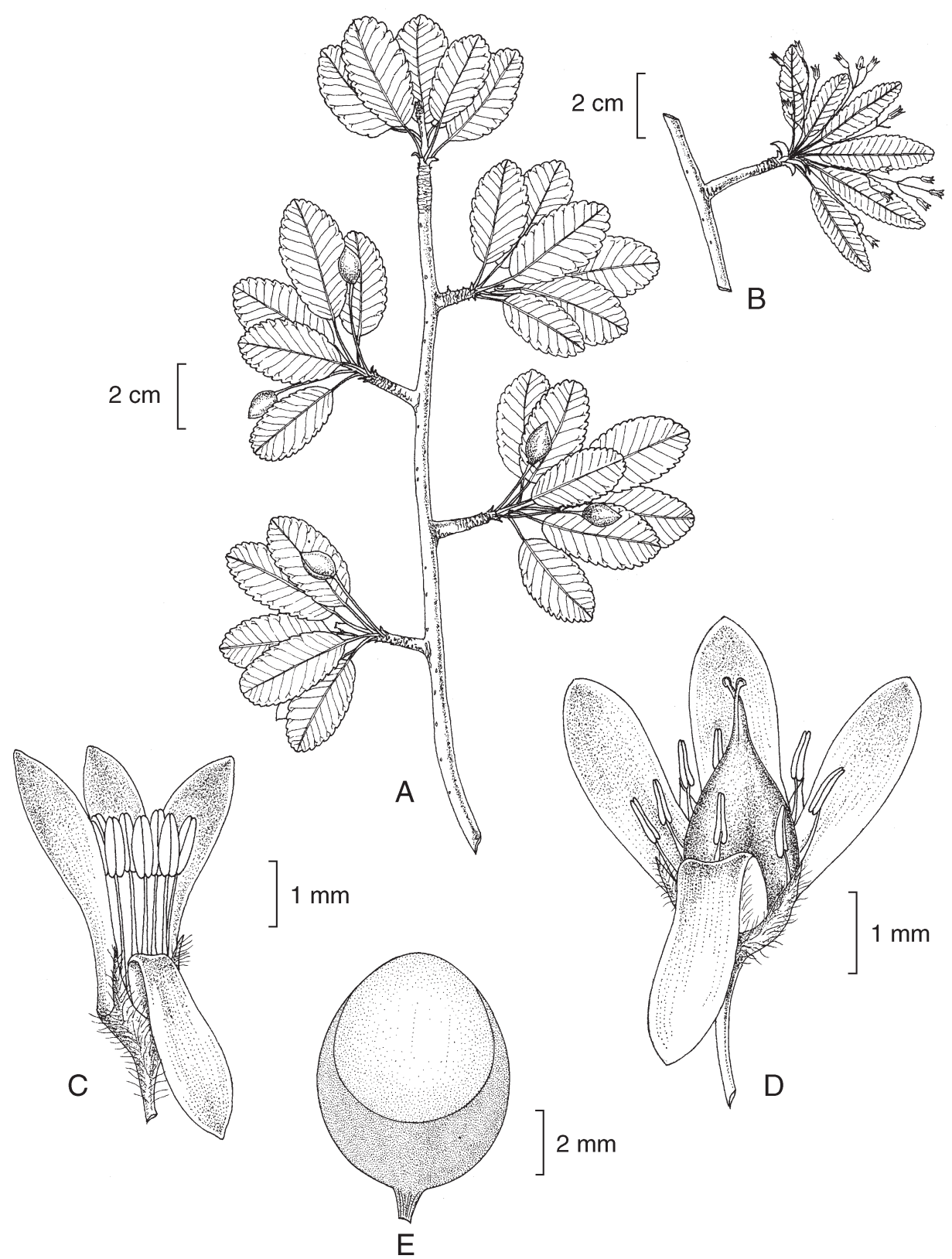

Fig. 2. Bursera simplex Rzed. \& Calderón. A. rama con hojas y frutos; B. ramilla con catafilos, hojas tiernas y flores masculinas; C. flor masculina; D. flor femenina; E. hueso. Ilustrado por Humberto Sánchez Córdova, excepto E que fue dibujado por Rogelio Cárdenas. 
la línea central, su ápice reflejo en la madurez, estambres 8, filamentos de ca. $2 \mathrm{~mm}$ de largo, anteras angostamente oblongas, de ca. $1 \mathrm{~mm}$ de largo, pistilodio diminuto; flores femeninas por lo general solitarias, con menos frecuencia en grupos de 3, sobre pedicelos filiformes hasta de $1.2 \mathrm{~cm}$ de largo (hasta de $4 \mathrm{~cm}$ en fruto), similares a las masculinas, aunque los pétalos a menudo sólo de ca. $3 \mathrm{~mm}$ de largo, estaminodios con anteras de ca. $0.6 \mathrm{~mm}$ de largo, ovario bilocular, glabro, estilo bífido; fruto bivalvado, elipsoide a obovoide, de 10 a $13 \mathrm{~mm}$ de largo, por lo general puntiagudo, rojizo en la madurez, glabro, hueso ampliamente elíptico a suborbicular, algo aplanado, de 6 a $7 \mathrm{~mm}$ de largo, de 4.5 a $5 \mathrm{~mm}$ de ancho, pseudoarilo de color rojo intenso, cubriendo menos de un tercio a cerca de la mitad, así como los cantos del hueso, la porción descubierta de color claro.

Tipo: México, Oaxaca, $4 \mathrm{~km}$ al E de San Juan Guegoyache, municipio de Totolapan, bosque tropical caducifolio, alt. $1100 \mathrm{~m}$, 9.VIII.1976, J. Rzedowski 34382 (IEB, isotipos por distribuirse).

Material adicional examinado: México, Oaxaca: $7 \mathrm{~km}$ al $\mathrm{S}$ de Totolapan, sobre la carretera a Zoquitlán, municipio de Totolapan, distrito de Tlacolula, $S$. Acosta 703 (IEB); $5 \mathrm{~km}$ al NW de Las Margaritas, municipio de Totolapan, $R$. Aguilar 240 (IEB); a $8 \mathrm{~km}$ al NO de Totolapan, carretera Tehuantepec a Oaxaca, distrito de Tlacolula, $R$. Cedillo Trigos y $R$. Torres 1572 (IEB, MEXU); $4 \mathrm{~km}$ al $\mathrm{W}$ de Totolapan, municipio de Totolapan, A. Flores 1356 (IEB); $9 \mathrm{~km}$ al W de Totolapan, municipio de Totolapan, G. Manzanero 1151 (IEB); 20 miles northwest of El Camarón (ca. 60 miles southeast of Oaxaca), R. McVaugh 21875 (ENCB); ca. 4 miles northeast of Totolapan (about 40 miles southeast of Oaxaca), $R$. McVaugh 21880 (ENCB); $4 \mathrm{~km}$ al NE de San Juanico, sobre el camino a Tehuantepec, municipio de Totolapan, J. Rzedowski 33033 (IEB), 33034 (IEB); $3 \mathrm{~km}$ al NE de San Juanico, municipio de Totolapan, J. Rzedowski 33599 (IEB); $2 \mathrm{~km}$ al N de La Ceiba, municipio de Totolapan, J. Rzedowski 34386 (IEB); 4 km al NW de Totolapan, sobre la carretera a Oaxaca, J. Rzedowski 35424 (IEB); alrededores de Totolapan, municipio de Totolapan, A. Saynes 1206 (IEB); $10 \mathrm{~km}$ desv. Portillo S. Dionisio - Totolapan, municipio de Totolapan, A. Saynes 1285 (IEB); along Hwy 190 about 13.6 mi. NW of San José de Gracia, W. D. Stevens et al. 2327 (IEB); El Derrumbadero, municipio de Totolapan, F. Ventura 16532 (IEB); cerca de Escuela Técnica Agropecuaria, municipio de Totolapan, $F$. Ventura 16576 (IEB); Mano de León, municipio de Zoquitlán, F. Ventura 16582 (IEB).

El área de Bursera simplex parece estar confinada a la porción media de la cuenca del río Tehuantepec, donde prospera en altitudes entre 850 y $1400 \mathrm{~m}$, siendo localmente una planta común y un componente importante del bosque tropical caducifolio. Florece en abril y mayo, al tiempo del surgimiento de las hojas. 
En función de sus flores tetrámeras y frutos bivalvados, la especie nueva pertenece a la sección Bullockia y está indudablemente relacionada con $B$. infernidialis Guevara \& Rzedowski, conocida de la porción occidental de la cuenca del Balsas, en Michoacán, Jalisco y Guerrero. Las principales diferencias entre estos dos taxa estriban en:

\begin{tabular}{|l|l|}
\hline \multicolumn{1}{|c|}{ Bursera infernidialis } & \multicolumn{1}{c|}{ Bursera simplex } \\
\hline $\begin{array}{l}\text { catafilos densamente pubescentes } \\
\text { en ambas caras }\end{array}$ & catafilos vilosos sólo en sus ápices \\
\hline hojas trifolioladas & hojas casi siempre simples \\
\hline inflorescencias masculinas & inflorescencias masculinas \\
paniculadas & racemosas \\
\hline frutos subesféricos, de 7 a $9 \mathrm{~mm}$ & frutos elipsoides a obovoides, \\
de largo & de 10 a 13 mm de largo \\
\hline $\begin{array}{l}\text { pseudoarilo cubriendo al menos } \\
\text { la mitad del hueso }\end{array}$ & pseudoarilo cubriendo menos de \\
la mitad del hueso
\end{tabular}

Otra planta posiblemente emparentada con B. simplex es B. biflora (Rose) Standl., conocida de Puebla y Oaxaca, común en la cuenca del Papaloapan, pero cuya área conocida penetra también a las porciones superiores de las cuencas del Balsas y del río Tehuantepec. En B. biflora la mayor parte de los individuos se caracteriza por sus hojas trifolioladas, pero tanto en Puebla como en Oaxaca se localizan varias poblaciones con todas sus plantas de hojas simples. Estas últimas pueden diferenciarse de B. simplex de la siguiente manera:

\begin{tabular}{|l|l|}
\hline \multicolumn{1}{|c|}{$\begin{array}{c}\text { Bursera biflora } \\
\text { (poblaciones con hojas unifoliadas) }\end{array}$} & \multicolumn{1}{c|}{ Bursera simplex } \\
\hline $\begin{array}{l}\text { catafilos lineares, densamente pubérulos } \\
\text { por fuera, glabros por dentro }\end{array}$ & $\begin{array}{l}\text { catafilos mayores oblongos, pilosos hacia } \\
\text { los ápices, glabros en la porción restante }\end{array}$ \\
\hline hojas de 1 a $3 \mathrm{~cm}$ de largo & hojas de 2 a $5 \mathrm{~cm}$ de largo \\
\hline pétalos de ca. $2 \mathrm{~mm}$ de largo & pétalos de ca. $5 \mathrm{~mm}$ de largo \\
\hline pedúnculos fructíferos filiformes & $\begin{array}{l}\text { pedúnculos fructíferos delgados } \\
\text { pero no filiformes }\end{array}$ \\
\hline frutos de 7 a 9 mm de largo & frutos de 10 a 13 mm de largo \\
\hline $\begin{array}{l}\text { pseudoarilo anaranjado, con la parte } \\
\text { expuesta del hueso negra }\end{array}$ & $\begin{array}{l}\text { pseudoarilo rojo oscuro, con la parte } \\
\text { expuesta del hueso de color claro }\end{array}$ \\
\hline
\end{tabular}


Otro elemento que se pronuncia también en favor de la existencia de una cercana relación entre $B$. simplex y $B$. biflora constituye el hecho de la existencia de la siguiente colecta procedente de un individuo estéril, posiblemente de origen híbrido, que presenta en la misma rama hojas simples y otras trifolioladas, en su mayoría de tamaño substancialmente mayor que las comúnmente encontradas en $B$. biflora:

México, Oaxaca: Portillo de San Dionisio, $13 \mathrm{~km}$ al SSE de Matatlán, alt. $1700 \mathrm{~m}$, matorrales secundarios, J. Rzedowski 34394 (IEB).

\section{AGRADECIMIENTOS}

Los autores agradecen al Biól. Miguel Angel Silva Farías su esfuerzo especial dedicado a la búsqueda y obtención de materiales de herbario de Bursera madrigalii. Dan las gracias asimismo a la Biól. Rosalinda Medina, quien tuvo la amabilidad de proporcionar información sobre ejemplares adicionales de las dos especies nuevas, depositadas en el herbario MEXU.

\section{LITERATURA CITADA}

Madrigal Sánchez, X. y L. I. Guridi Gómez. 2002. Los árboles silvestres del municipio de Morelia, Michoacán. México. Ciencia Nicolaita 33: 29-55. 